\title{
Epithelioid Sarcoma: A Case Report
}

\author{
Pooja Jain* and Ranjini Kudwa
}

Department of Pathology, Kasturba Medical College Manipal, Manipal, Karnataka, India

\begin{abstract}
Epithelioid sarcoma is a rare highly aggressive soft tissue sarcoma with a high incidence of local recurrence and metastasis. It is a tumor of uncertain histogenesis \&poses a diagnostic difficulty as it is often misdiagnosed as other benign or malignant condition. We present the case of 49-year-old malepresenting with chief complaints of pain and gradually progressive swelling on right 3 rd toe since 3 months. Based on clinicoradiological evaluation suggestive of malignancy, an amputation of toe was done. Histopathological \& Immunohistochemical findings suggestive of Epithelioid sarcoma. The patient was referred to the oncology centre for further management where he was advised wide excision followed by radiotherapy. No regional or distant metastasis was found.
\end{abstract}

Keywords: Rare, Epithelioid Sarcoma, Aggressive

\section{Introduction}

Epithelioid sarcoma (ES) is an uncommon slow growing soft tissue sarcoma with a poor long term prognosis .It account for less than $1 \%$ of all soft tissue sarcomas. It is a rare mesenchymal tumor of unknown histogenesis, display multidirectional differentiation which is predominantly epithelial $^{(5)}$ Usually occurs in the distal extremities of young adults as a as a subcutaneous or deep dermal mass. It has a strong propensity for local recurrence and, ultimately, metastasis. This tumor poses diagnostic difficulties both clinically \& histologically, resulting in a high degree of misdiagnosis.

\section{Case Report}

A 49 year old male presented with complaints of pain and gradually progressive swelling on right 3rd toe since 3 months. Insidious onset with no history of trauma or fever and other constitutional symptoms. On examination $7 \times 4 \mathrm{~cm}$ swelling was present on the dorsum of 3rd toe, and diffuse swelling on plantar aspect of sole. No distal neurovascular deficit present \& movement of all toes were normal. On examination $7 \mathrm{x} 4 \mathrm{~cm}$ swelling was present on the dorsum of 3rd toe, and diffuse swelling on plantar aspect of sole. Based on clinico-radiological evaluation suggestive of malignancy, an amputation of toe was done.

On gross examination right $3^{\text {rd }}$ toe with attached metatarsal showed a grey white irregular growth measuring $3.5 \times 3 \times 3$ $\mathrm{cm}$ on dorsal surface along with grey black areas on skin. cut section showed grey white areas and specks of haemorrhage (Fig.1.)

Histopathological examination showed a tumor involving deep dermis and extending into subcutaneous tissue composed of lobules and nests of large polygonal cells with eosinophilic cytoplasm, vesicular nuclei, prominent nucleoli, central necrosis and myxoid degeneration in a collagenised stroma with numerous eosinophils and few plasma cells (Fig.2,3,4,5). Foci of spindle cells arranged in vague whorls with mitotic figures with intervening collagen bundles in a stroma with myxoid degeneration noted.

\section{Gross appearance;}

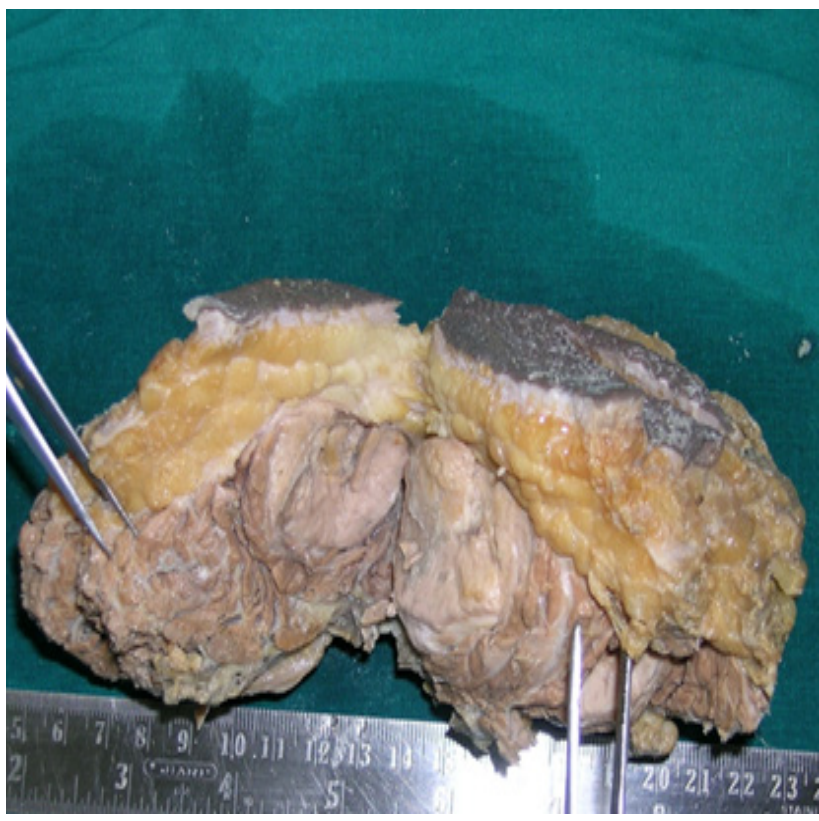

Fig. 1: Toe with attached metatarsal : dorsal surface showed a grey white irregular growth. 


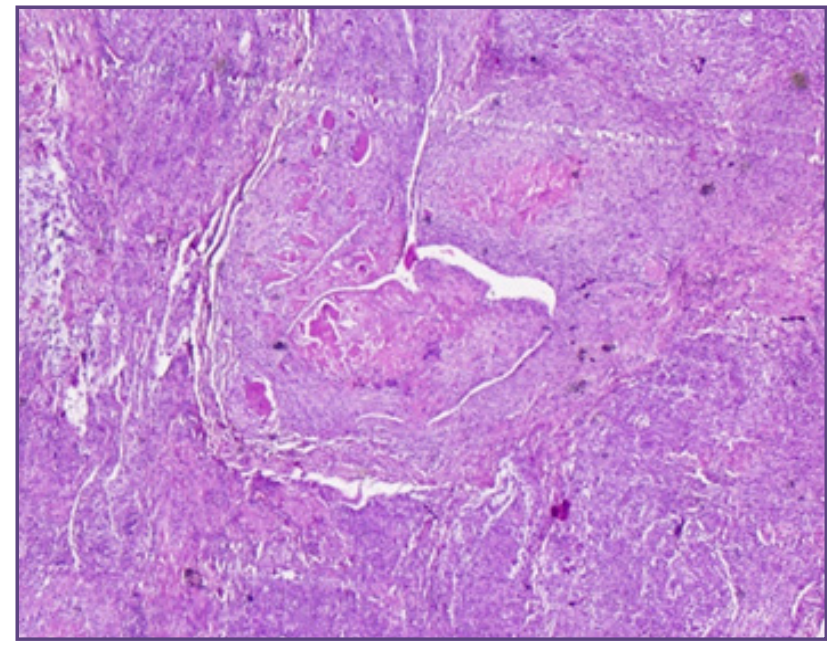

Fig. 2: The tumor is composed of lobules \& nests of large round or polygonal epithelioid cells in a pseudogranulomatous pattern with areas of necrosis(H\&E, 10x).

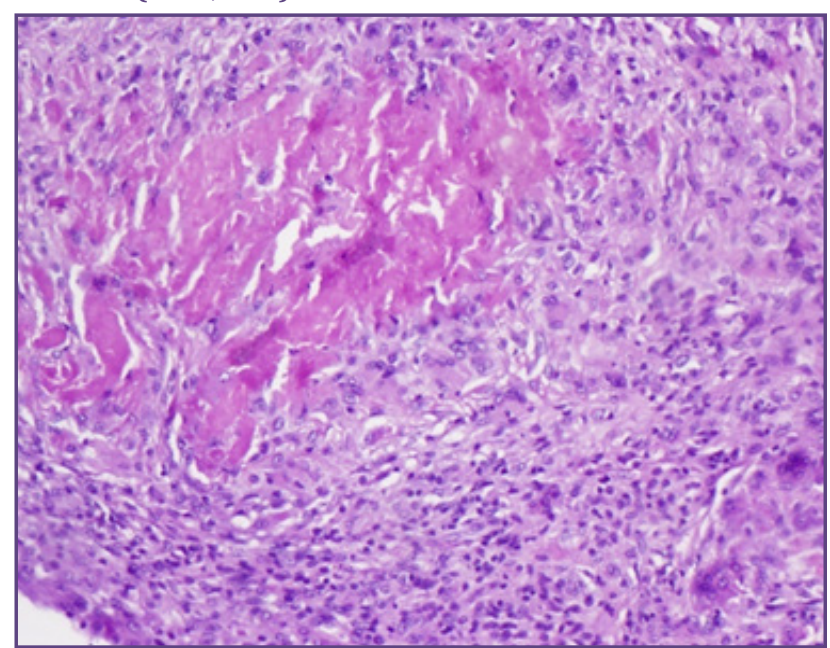

Fig. 4: High power view showing granulomalike growth pattern with central necrosis and a mixture of spindled and epithelioid cells surrounding necrotic areas(H\&E, 100x).

\section{Immunohistochemical findings}

On immunohistochemical examination cells were found diffusely positive for cytokeratin, Vimentin \& focally positive for epithelial membrane antigen. (Fig:6,7,8).

Histopathological \& Immunohistochemical findings suggestive of Epithelioid sarcoma. The patient was referred to the oncology centre for further management where he was advised wide excision followed by radiotherapy. No regional or distant metastasis was found.

\section{Discussion}

Epithelioid sarcoma is a soft-tissue tumor, composed of large, polygonal cells resembling carcinomas, was first

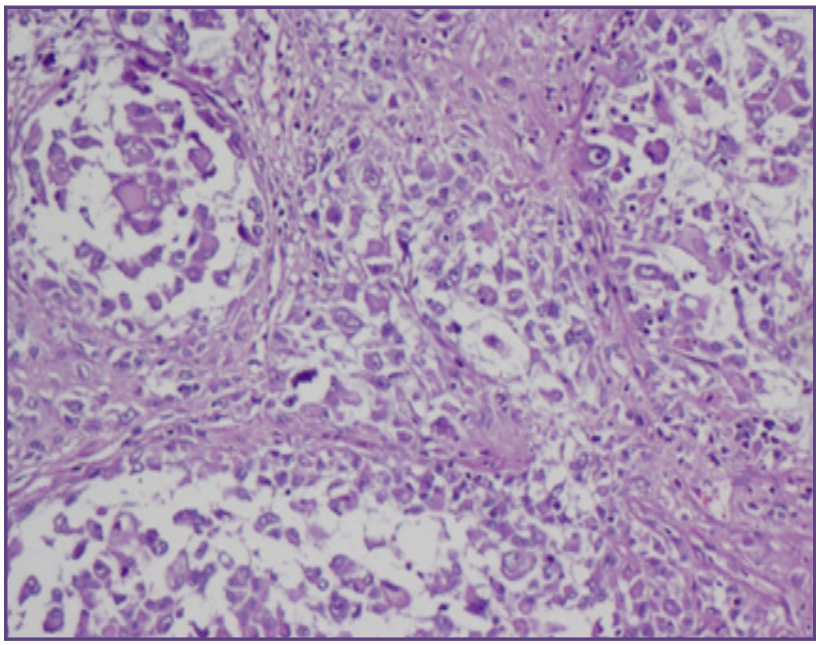

Fig. 3: Tumor cells have abundant eosinophilic cytoplasm, vesicular nuclei and prominent central prominent nuclei (H\&E, 100x).

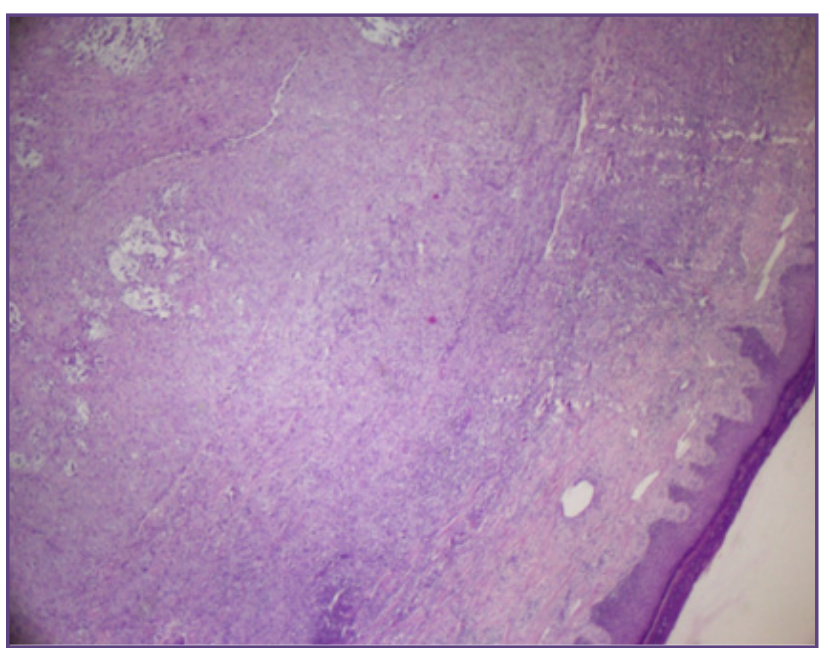

FIG. 5: Low power view showing tumor involving deep dermis and extending into soft tissues(H\&E, 10x).

described by Enzinger in $1970 .{ }^{[1]}$ It is a relatively rare, soft-tissue sarcoma of unknown histogenesis, usually slowgrowing throughout a period of years, with a seemingly benign morphologic appearance and is, therefore, often misdiagnosed on first encounter. ${ }^{[2]}$ As on histology, it present as typical "granuloma-like" appearance of tumor cells surrounding a necrotic focus. ${ }^{[1,2]}$ In its conventional or classic form, it is usually a solitary or multifocal tumor involving the dermis, subcutis, or deeper soft tissues in the distal extremities of young adults in $55 \%$ to $60 \%$ of cases. ${ }^{[2,3]}$

Diagnosis is made by typical histological features of distinct nodular aggregates of epithelioid and spindle cells with zonal necrosis ${ }^{[2,3]}$ Epithelioid sarcoma is a 


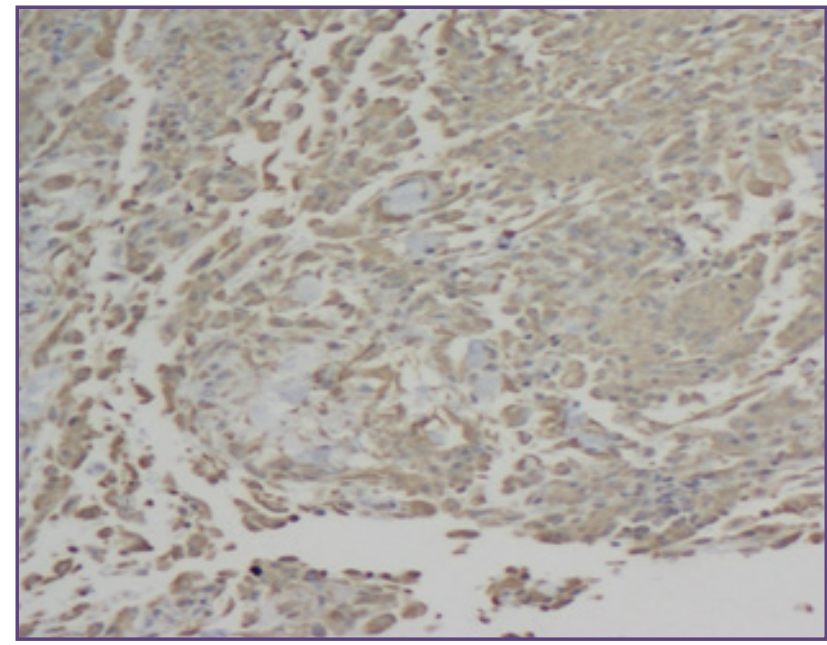

Fig. 6: IHC--Vimentin.

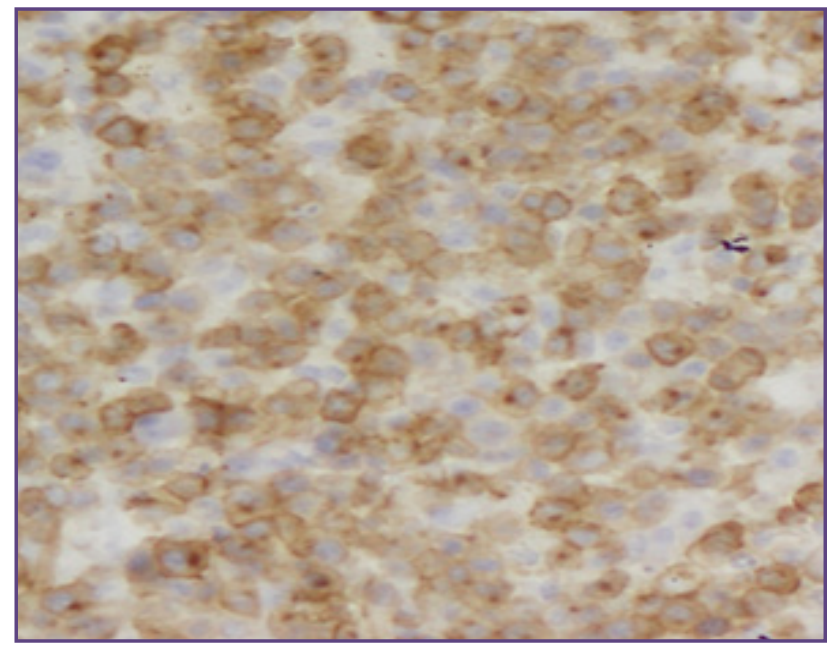

Fig. 7: IHC--CK.

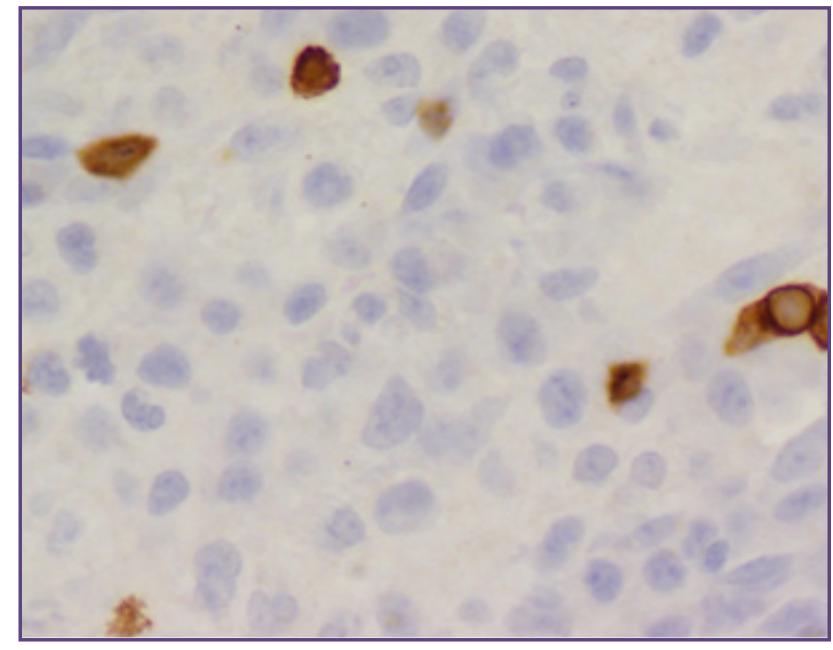

Fig. 8: IHC--EMA. mesenchymal tumor with a predominant epithelial differentiation, showing reactivity for both epithelial and mesenchymal markers, such as cytokeratin, epithelial membrane antigen, vimentin and CD34. ${ }^{[4,2,6]}$ Present case has typical histological features \& shows reactivity with both epithelial and mesenchymal markers.

Epithelioid sarcoma has a strong tendency to develop local recurrences and metastasis thereafter to regional lymph nodes, lung, bone, brain, noted in $40 \%$ to $50 \%$ of cases and can be reduced by adequate surgery. ${ }^{[4,2,5]}$

Epitheloid sarcoma is often misinterpreted on histopathology and misdiagnosed as granulomatous disease, melanoma, synovial sarcoma, ulcerating squamous cell carcinoma, epithelioid hemangioendothelioma.

However, in epithelioid sarcoma, the epithelioid cells are more sharply defined than granulomas the epithelioid cells are larger, more eosinophilic and less mature in nature and the presence of mitotic activity. ${ }^{[4]}$

should raise the index of suspicion for ES, as well as the presence of diffuse epithelial marker positivity indicates the correct diagnosis Epithelioid sarcoma can be differentiated from synovial sarcoma by the absence of pseudoglandular structures and, tendency to ulcerate. Epitheliod hemangioendothelioma is a low-grade vascular neoplasm which can mimic epithelioid sarcoma and can be excluded by the absence of necrosis \& low mitosis. ${ }^{[5]}$

Prognosis is dependent on the depth of the tumor in relation to the deep fascia, local recurrence and regional lymph node The present case did not show local spread or involvement of regional lymph nodes ${ }^{[6]}$ Distant metastatic disease has been reported in up to $45 \%$ of patients with epithelioid sarcoma. ${ }^{[1,7]}$ The metastasis is usually to the lung and pleura. ${ }^{[7]}$ No distant metastasis was detected in our patient at the referred oncology centre.

The treatment of primary disease is wide local excision, followed by adjuvant radiotherapy to lower the risk of local recurrence. ${ }^{[8]}$ However, Amputation is required relatively frequently in epithelioid sarcoma because of multifocal disease, but there is no survival advantage from primary amputation. Chemotherapy is recommended for metastatic disease with standard regimen. ${ }^{[8]}$

\section{Conclusion}

ES is a rare, slow growing, high-grade, soft tissue tumor with an inherent propensity for local recurrence, regional lymph node involvement, and distant metastases. This tumor is often misdiagnosed as benign process because 
of its Innocuous presentation. Hence, clinician must be aware to avoid misdiagnosis. Unusual soft tissue nodules on distal extremities of young adult should be thoroughly investigated if malignant behavior is suspected due to aggressive behavior of this entity. Our patient underwent wide excision followed by radiotherapy after diagnosis and no regional or distant metastasis was found.

\section{Refrences}

1. Enzinger FM. Epithelioid sarcoma: a sarcoma simulating a granuloma or a carcinoma. Cancer. 1970;26:1029-1040

2. Rekhi, B., Gorad, B.D. \& Chinoy, R.F. Proximal-type epithelioid sarcoma - a rare, aggressive subtype of epithelioid sarcoma presenting as a recurrent perineal mass in a middle- aged male. World J Surg Onc 5, 28 (2007) doi:10.1186/1477-7819-5-28

3. Laila C, Louis G, Philippe T, Anne V D, Fleur G, Marie JTL, Dominique R, Yves M R, Françoise C, Paul F, Jean
M C, Epithelioid Sarcoma: A Clinicopathologic and Immunohistochemical Analysis of 106 Cases From the French Sarcoma Group, American Journal of Clinical Pathology, Volume 131, February 2009, Pages 222-227.

4. Weiss SW, Goldblum JR. Malignant soft tissue tumors of uncertain type. In: Enzinger FM, Weiss SW, editors. Soft tissue Tumors. 4th ed. St Louis: Mosby; 2001. p. 1521-38.

5. Billings SD, Folpe AL, Weiss SW. Epithelioid sarcoma-like hemangioendothelioma. Am J Surg Pathol 2003;27:48-57.

6. Spillane AJ, Thomas JM, Fisher C. Epithelioid sarcoma: The clinicopathologic complexities of this rare soft tissue sarcoma. Ann Surg Oncol 2000; 7:218-25

7. Ross HM, Lewis JJ, Woodruff JM, Brennan MF. Epithelioid sarcoma: Clinical behavior and prognostic factors of survival. Ann Surg Oncol 1997;4:491-5.

8. Miettinen et al.: Epithelioid sarcoma: An immunohistochemical analysis of 112 classical and variant cases and a discussion of the differential diagnosis. Human Pathology Volume 30, Issue 8, Pages 934-942. Aug 1999.

*Corresponding author:

Dr. pooja Jain, 463, Sonia girls hostel, KMC, Manipal, Karnataka, India

Phone: +91 9999352553

Email: drpoojajain83@gmail.com,

Financial or other Competing Interests: None. 